Laurence lan Tan, MD

Jose Florencio F. Lapeña, Jr., MA, MD 1,2

'Department of Otorhinolaryngology

Philippine General Hospital

University of the Philippines Manila

2Department of Otorhinolaryngology

UP College of Medicine-Philippine General Hospital

University of the Philippines Manila
Correspondence: Jose Florencio F. Lapeña, Jr. MA, MD

Department of Otorhinolaryngology

Ward 10, Philippine General Hospital

Taft Avenue, Ermita, Manila 1000

Philippines

Phone: (632) 5264360

Fax: (632) 5255444

Email: lapenajf@upm.edu.ph

Reprints will not be available from the author.

No funding support was received for this study. The authors signed a disclosure that they have no proprietary or financial interests with any organization that may have a direct interest in the subject matter of this manuscript, or in any product used or cited in this report.

\title{
A 41-year-old Female Presenting with Sicca Syndrome
}

\section{Methods: \\ Design: Case report \\ Setting: Private clinic \\ Patient: One}

Objective: To describe a case of Sicca syndrome, review the literature on and discuss the diagnostic criteria for Sjögren Syndrome.

Results: A 41-year-old female presenting with throat dryness, dry eyes, bilateral parotid enlargement, vaginal dryness and multiple joint pains previously seen by four specialists over three decades was finally diagnosed with Sjögren syndrome.

Conclusion: Sjögren syndrome presents uncommonly. As the presentation and clinical course should be taken as a whole, and no single test can be considered as a gold standard for its diagnosis, the disease entity may actually be under diagnosed, misdiagnosed or frequently present as diagnostic dilemmas. A comprehensive history and physical examination beyond the usual focus of the otorhinolaryngologic evaluation is the key for the astute clinician. Our experience may indicate a need for further evaluation of the EU revised criteria for Sjögren syndrome classification in the local setting.

Key words: sicca syndrome, sjogren syndrome, keratoconjunctivitis sicca, stomatitis sicca, xerostomia, dysparenuia

Intially termed keratoconjuctivitis sicca by the ophthalmologist Henrik Sjögren in the 1930's, Sicca syndrome currently includes dry eyes, decreased lacrimation, dry mouth and decreased salivation, which can be the result of a variety of underlying causes.'

Sicca ranks among the more frequently encountered symptoms in otorhinolaryngology with Sjögren syndrome as one of the many systemic conditions that present in the head and neck region. Often patients come into the clinic with a variety of non specific complaints, making diagnosis elusive and treatment even more difficult. Criteria for diagnosis are evolving, although treatment remains symptomatic. We present the case of a frustrated lady whose condition was undiagnosed by four different physicians over three decades. 
CASE REPORTS

\section{CASE REPORT}

A 41-year-old female from San Pablo City was referred for throat dryness of twenty-six years duration, unrelieved by a thyroidectomy (!) recommended by another physician. On review, she had recurrent bilateral parotid swelling since childhood which had been managed with such home remedies as topical vinegar or gentian violet applications. No consults were made, as her parents dismissed the condition as mumps.

Five years before consultation, she started to note decreased tears, dry eyes and pain in the tempomandibular area with recurrence of bilateral parotid enlargement. Throat dryness also persisted, and was now associated with dysphagia and odynophagia for hard solids. Another otorhinolaryngologist considered a parotid tumor and advised a sialogram which she deferred, opting instead for complementary and alternative herbal medications with slight relief of throat dryness.

Her symptoms persisted until two months prior to consult, when she started to experience non-migratory multiple- joint pains. She consulted another physician who referred her to the senior author.

On review, she also admitted chronic vaginal dryness and dyspareniua. She had no headache, dizziness or sensorial changes, no vomiting, epigastric pain, rash, oliguria, uremic fetor or chest pain. She had no history of radiotherapy, jaundice, hepatitis or atopy and denied any chronic use of medication.

Aside from the thyroidectomy in 1980, she had two caesarian sections in 1984 and 1986; a laparotomy and excision of ruptured ovarian cyst in 1989; and total abdominal hysterectomy and bilateral salpingooophorectomy in 1999. A housewife, she had no exposure to radiation, heavy metals or chemicals and did not use tobacco or ethanol. Her family medical history did not include atopic or autoimmune disease, cancer, renal disease or hepatitis.

Physical examination revealed keratoconjunctivitis sicca, xerostomia with no salivary flow, a fissured tongue with flattened papillae, multiple dental carries, vague enlargement of both parotid glands and a healed transverse cervical incision. The rest of the ear, nose, throat, head and neck findings were unremarkable. Schrimer's Test without anesthesia confirmed complete absence of tears (Figure 1).

With a presumptive diagnosis of Sjögren Syndrome, she was started on artificial tears (Tears Naturale ${ }^{T M}$, Alcon Lab Phils San Juan, Metro Manila) and saliva (bioXtra ${ }^{\mathrm{TM}}$, Bio-X Healthcare 5032 Les Isnes, Belgium) and a labial biopsy was recommended. She was also referred to a dentist and to a rheumatologist who requested ANA, anti SS-A and anti SS-B tests. Relieved to have a diagnosis, she opted not to have these tests done. Interestingly, a confirmatory thyroid ultrasonogram revealed normal thyroid despite the previous surgery and thyroid function tests were all normal.

\section{DISCUSSION}

Sicca syndrome can result from a variety of underlying causes, with connective tissue and autoimmune disorders such as Systemic Lupus Erythematosus (SLE), Rheumatoid Arthritis (RA) and Sjögren's Syndrome (SS) among the primary differentials. SLE commonly presents with cutaneous lesions-malar rash, discoid rash, and oral ulcers while RA prominently features migratory polyarthritis. Rheumatoid Factor (RF) is positive in most cases of RA. In addition, more than $50 \%$ of patients with high levels of RF in their blood have SS. Many patients with RA also have SS. There is sexual predilection for both diseases, with two to three times as many women as men having RA, and $90 \%$ of $\mathrm{SS}$ cases involving females.'

The diagnosis of Sjögren syndrome poses difficulty as no single clinical symptom, sign or lab test can be regarded as confirmatory or the gold standard. ${ }^{2}$ None of the proposed diagnostic and classification criteria were widely accepted until 1993, when the European criteria gained comparatively more consensus among textbooks, experts and clinicians. ${ }^{2,3,4}$ The current internationally accepted classification criteria for Sjögren syndrome is a revised version of the European criteria proposed by the American-European Consensus Group in 2002. ${ }^{4}$ [Appendix]

Our patient met four of the six criteria for diagnosis of primary SS: Item I, ocular symptoms (foreign body sensation, eye irritation, redness); Item II, oral symptoms (dry mouth, swollen salivary glands); Item III, a Schirmer's I test, performed without anesthesia $(<5 \mathrm{~mm}$ in $5 \mathrm{~min})$ and Item $\mathrm{V}$, unstimulated salivary flow $(<1.5 \mathrm{ml} / 15 \mathrm{mins})$.

The presence of any four of the six items is indicative of Primary SS as long as either item IV (Histopathology) or VI (Serology) is positive. Unfortunately, both were declined by our patient. The presence of any 3 of the 4 objective criteria items (items III, IV, V, VI) or the classification tree procedure represent valid alternative methods for classification. Our patient only fulfilled 2 of the 4 objective criteria items, III and V.

After having seen four physicians without a diagnosis, would our diagnosis now be futile on the basis of inadequate criteria?

A study by $\mathrm{Zhao}^{5}$ found the American-European criteria for diagnosis of Sjögren syndrome to have a high sensitivity and specificity for primary SS patients in China, but questioned the value of histology (lip biopsy) included in EU-Am criteria. They found that even without lower lip minor salivary gland histopathology results, these criteria could reach a sensitivity of $87.2 \%$ vs $89.2 \%$ with a lip biopsy, which they determined to have no significant difference. ${ }^{5}$ Our patient has fulfilled four of the six criteria allowing a diagnosis of primary Sjögren syndrome with $97.4 \%$ sensitivity and $89.4 \%$ specificity. ${ }^{2}$

What sensitivity and specificity levels are acceptable to make a diagnosis of primary Sjögren syndrome, knowing that the diagnosis entails a 40-fold increase in the risk of developing lymphoma, and that treatment consists in palliation of symptoms? Are criteria with neither local data nor local prevalence studies applicable locally?

Our patient opted not to undergo any further tests despite full education on the increased risk of lymphoma for Sjögren syndrome and the possibility of misdiagnosis. Positive serologic or histological results will not alter immediate management, and treatment is directed mainly at symptom palliation and early detection of malignancy or life threatening complications. Keratoconjunctivitis sicca is usually treated with artificial tears (as given to our patient), steroid and non-steroid eye drops, tear passage occlusion or soft adherent lenses. Stomatitis sicca may sometimes be sufficiently relieved by $\mathrm{N}$-acetyl-cysteine, artificial saliva (given to our patient), chewing gum and B-vitamin complex. 
Salivary gland enlargement and extra glandular signs are often of less concern but can be treated by NSAIDs and corticosteroids. Her quality of life has dramatically improved, not so much because of the supportive therapy instituted, but because she now has a name for, and an understanding of her disease.

Sjögren syndrome presents uncommonly. As the presentation and clinical course should be taken as a whole, and no single test can be considered as a gold standard for its diagnosis, the disease entity may actually be under diagnosed, misdiagnosed or frequently present as diagnostic dilemmas. A comprehensivehistoryand physical examination beyond the usual focus of the otorhinolaryngologic evaluation is the key for the astute clinician. Our experience may indicate a need for further evaluation of the EU revised criteria for Sjögren syndrome classification in the local setting.

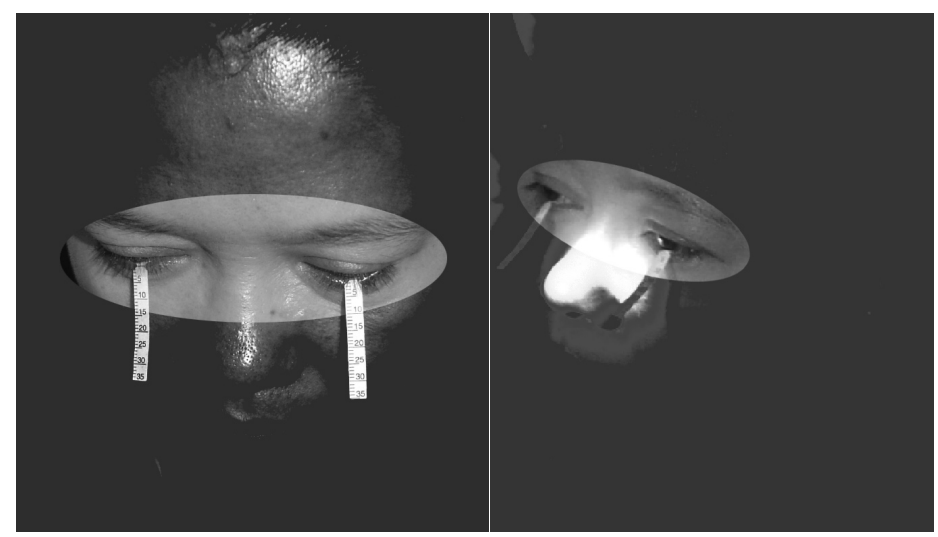

Figure 1. Schrimer's Test showing absence of production of tears.

\section{APPENDIX}

\begin{tabular}{l|l}
\multicolumn{2}{c}{ Criteria for diagnosis of Sjögren's syndrome $^{2}$} \\
\hline Primary SS Criteria & Secondary SS Criteria \\
\hline $\begin{array}{l}\text { any } 4 \text { of the } 6 \text { items with either item IV } \\
\text { or VI is positive }\end{array}$ & $\begin{array}{l}\text { the presence of item I or item II plus any } 2 \text { from } \\
\text { among items III, IV, and V }\end{array}$ \\
\hline Any 3 of the 4 objective criteria & \\
\hline Classification tree procedure & \\
\hline
\end{tabular}

\section{Classification criteria for Sjögren's syndrome (2002) ${ }^{2}$}

1. Have you had daily, persistent, troublesome dry eyes for more than 3 months?

2. Do you have a recurrent sensation of sand or gravel in the eyes

3. Do you use tear substitute more than 3 times a day?

1. Have you had daily feeling of dry mouth for more than 3 months?

2. Have you had a recurrent persistently swollen salivary glands as an adult?

3. Do you frequently drink liquids to aid in swallowing dry food?

1. Schirmer's I test, performed without anaesthesia ( $\leq 5 \mathrm{~mm}$ in $5 \mathrm{~min})$

2.Rose bengal score or other ocular dye score ( $\geq 4$ according to van Bijsterveld's scoring system)

Histopathology: In minor salivary glands (obtained through normal-appearing mucosa) focal lymphocytic sialoadenitis, evaluated by an expert histopathologist, with a focus score $\geq 1$, defined as a number of lymphocytic foci (which are adjacent to normal-appearing mucous acin and contain more than 50 lymphocytes) per $4 \mathrm{~mm} 2$ of glandular tissue1

1. Unstimulated whole salivary flow ( $\leq 1,5 \mathrm{ml}$ in $15 \mathrm{~min}$ )

2. Parotid sialography showing the presence of diffuse sialectasias (punctate, cavitary or destructive pattern), without evidence of obstruction in the major ducts

3. Salivary scintigraphy showing delayed uptake, reduced concentration and/or delayed excretion of tracer

\section{Autoantibodies: presence in the serum of the Antibodies to Ro(SSA) or La(SSB) antigens, or both}

Exclusion Criteria

Past head and neck radiation treatment

Hepatitis C infection

Acquired immunodeficiency disease (AIDS)

Pre-existing lymphoma

Sarcoidosis

Graft versus host disease

Use of anticholinergic drugs (since a time shorter than 4-fold the half life of the drug

\section{REFERENCES}

1. AACC.org [homepage on the Internet]. Washington DC. American Association for Clinical Chemistry 2001-2006 Available from http://www.aacc.org/.

2. Vitali C, Bombadieri S, Jonsson R Classification criteria for Sjögren's syndrome: a revised version of the European criteria proposed by the American-European Consensus Group. Ann Rheum Dis 2002;61, 554-8.

3. Vitali C, Bombardieri S, Moutsopoulos HM, Alexander EL, Carsons SE, Daniels TE, et al. Assessment of the European classification criteria for Sjögren's syndrome in a series of clinically defined cases. Results of a prospective multicentre study. Ann Rheum Dis 1996; 55, 116-21.

4. Zhao Y, Zheng W, Zhou W, Zeng X, Zhang F, Tang F, Dong Y The evaluation of the international classification criteria (2002) for primary Sjögren's syndrome in Chinese patients. APLAR J Rheum 2005; 8 (3), 184-187.

5. Zhang ZL, Dong Y Clinical and immunological features of primary Sjögren's syndrome with liver damage: 30 cases of clinical analysis. Chin J Rheum 1998; 2, 92-6. 